\title{
Prediction of right ventricular dysfunction from radiographic estimates of right descending pulmonary artery in hemodynamically stable pulmonary embolism patients
}

\author{
Yasin $\mathrm{Abul}^{1}$, Savas Ozsu ${ }^{1}$, Sait Karakurt², Beste Ozben ${ }^{3}$, Ismet Durmus ${ }^{4}$, \\ Ahmet Toprak ${ }^{5}$, Tevfik Ozlu¹, Turgay Celikel ${ }^{2}$
}

${ }^{1}$ Karadeniz Technical University, Faculty of Medicine, Department of Pulmonary Medicine, Trabzon, Turkey ${ }^{2}$ Marmara University, Faculty of Medicine, Department of Pulmonary and Critical Care, Istanbul, Turkey

${ }^{3}$ Marmara University, Faculty of Medicine, Department of Cardiology, Istanbul, Turkey ${ }^{4}$ Karadeniz Technical University, Faculty of Medicine, Department of Cardiology, Trabzon, Turkey

${ }^{5}$ Tulane Center for Cardiovascular Health, New Orleans, USA

\begin{abstract}
Background: The evaluation of right ventricular $(R V)$ dysfunction by echocardiography is one of the most important established determinants of the prognosis of acute pulmonary embolism. The aim of the study was to investigate possible association between diameter of right descending pulmonary artery on chest $X$-rays and $R V$ dysfunction by echocardiography in hemodynamically stable pulmonary embolism patients.

Methods: Eighty-nine patients with the diagnosis of hemodynamically stable pulmonary embolism were included.

Results: The frequency of $R V$ dysfunction was significantly higher in patients with an enlarged right descending pulmonary artery on chest $X$-rays $(p=0.001)$. There was a significant positive correlation between the diameter of the right descending pulmonary artery on postero-anterior chest $X$-rays and the diameter of the $R V(r=0.469 ; p=0.002)$. Diameter of right descending pulmonary artery on chest $X$-rays was also found as a significant predictor of $R V d y$ sfunction besides the troponin-T levels and systolic pulmonary arterial pressure $(p<0.05)$.

Conclusions: Diameter of right descending pulmonary artery on chest X-rays may provide information about the risk for pulmonary embolism patients and may be used as a prognostic radiological parameter for the appropriate management of acute pulmonary embolism. (Cardiol J 2013; 20, 2: 184-189)
\end{abstract}

Key words: pulmonary embolism, right ventricular dysfunction

\section{Introduction}

The evaluation of right ventricular (RV) dysfunction by echocardiography is one of the most important established determinants of the pro- gnosis of acute pulmonary embolism (PE) [1,2]. Mortality has been known to be high in patients with RV dysfunction at presentation [3]. However echocardiography is a user-dependent procedure and is not always readily available for a diagnostic

Address for correspondence: Yasin Abul, Assist. Prof., MD, Karadeniz Teknik University Farabi Hastanesi Gogus Hastaliklari A.D. Trabzon, Turkey, tel: +90 536 5268765, fax: +90 212 2589943, e-mail: abulyasin@yahoo.com 
workup and risk stratification of PE. Recently many markers including cardiac troponin I or T $[4,5]$, N-terminal pro-BNP (NT-proBNP) [6], B-type natriuretic peptide (BNP) [7], the plasma heart-type fatty acid binding protein [8], growth differentiation factor (GDF)-15 [9] and the C-reactive protein [10] have been shown to be associated with an increased risk of complications and death in patients suffering acute PE. These markers have also been shown to be associated with RV dysfunction which is a gold standard measure of the prognosis for PE patients. However these biomarkers may not be easily accessible in clinical settings due to the high expenditure involved or limited availability of them. Diagnostic methods that are easily applied and accessible are needed to determine RV dysfunction in PE for accurate and rapid risk stratification.

Radiographic abnormalities have been shown to be associated with PE [11-13]. Transient enlargement of right descending pulmonary artery in PE patients has been reported in various studies [12, 14-16]. Previous reports have shown a correlation between right descending pulmonary artery diameter and systolic pulmonary artery pressure estimated by echocardiography [17] and it has also been shown that a diagnosis of pulmonary hypertension can be derived from radiographic estimates of pulmonary arterial size [18]. An association between enlargement of the right descending pulmonary artery and RV hypokinesis has been previously studied but other RV parameters of dysfunction, including dilatation of the RV (diastolic diameter $>30 \mathrm{~mm}$ ), abnormal motion of the interventricular septum, hypokinesis of the RV, or tricuspid valve regurgitation (jet velocity $>2.5 \mathrm{~m} / \mathrm{s}$ ) and their association with enlargement of the right descending pulmonary artery on chest X-rays have not been studied. We designed the present study in order to investigate the possible prediction of $\mathrm{RV}$ dysfunction from radiographic estimates of right descending pulmonary artery in PE patients.

\section{Methods}

The investigation conforms to the principles outlined in the Declaration of Helsinki. The study was a retrospective chart review approved by the local ethics committee. All the radiological images and echocardiographical measurements were obtained from the hospital database sysytem. Eighty-nine patients with a diagnosis of hemodynamically stable $\mathrm{PE}$ were included (M:F $=41 / 48$; age $65 \pm 13.5$ ) in the study. PE patients without ha- ving RV echocardiographic measurements within $24 \mathrm{~h}$ of admission were not included into the study. $\mathrm{PE}$ patients with techinical difficulties about reading of postero-anterior (PA) chest X-rays were not included into the study. The patients with pre-existing severe heart failure or mitral valve regurgitation or right myocardial infarction and with pre-existing severe chronic obstructive pulmonary disease interfering both RV echocardiographic measurements and right descending pulmonary artery on chest $\mathrm{X}$-rays were also not included into the study population. Diameter of right descending pulmonary artery on PA chest X-ray on admission was measured by a at least two physician trained by a expert radiologist for the measurement of right descending pulmonary artery on PA chest X-ray. Physicians measuring diameter of right descending pulmonary artery on PA chest X-ray on admission were blinded to the result of measurement for RV dysfunction on echocardiography.

The diagnosis of PE was made by using spiral computerized tomography-angiography or a ventilation-perfusion scan with clinical probability score in all patients. The RV function was evaluated by conventional transthoracic echocardiography and $\mathrm{RV}$ dysfunction was defined if at least one of the following was present: 1 - dilatation of the $\mathrm{RV}$ (diastolic diameter $>30 \mathrm{~mm}$ ), $2-$ abnormal motion of the interventricular septum, 3 - hypokinesis of $\mathrm{RV}$, or 4 - tricuspid valve regurgitation (jet velocity $>2.5 \mathrm{~m} / \mathrm{s}$ ) [19].

Chest X-ray films (standard PA view) of each subject were taken on admission to the hospital with the diagnosis of PE. The diameter of the right descending pulmonary artery on PA chest X-ray was measured according to previously reported methods [20,21] defining the normal upper limit as $15 \mathrm{~mm}$ for females and $16 \mathrm{~mm}$ for males (Fig. 1). If this measure was $16 \mathrm{~mm}$ or greater, or $17 \mathrm{~mm}$ or greater, for females and males respectively, the right descending pulmonary artery was defined as enlarged.

Patients who had been diagnosed with PE received standard anticoagulant treatment which was either low molecular weight heparin (LMWH, particularly enoxaparin: $100 \mathrm{IU} / \mathrm{kg}$ twice daily) or standart unfractionated heparin which is a bolus of $80 \mathrm{U} / \mathrm{kg}$ followed by a continuous infusion of $18 \mathrm{U} / \mathrm{kg} / \mathrm{h}$ and targeting activated partial thromboplastin time by a factor of $1.5-2.5$. Warfarin was given for a period of at least 3 months with the aim of, reaching an international normalized ratio (INR) of 2.0-3.0 during follow-up. 


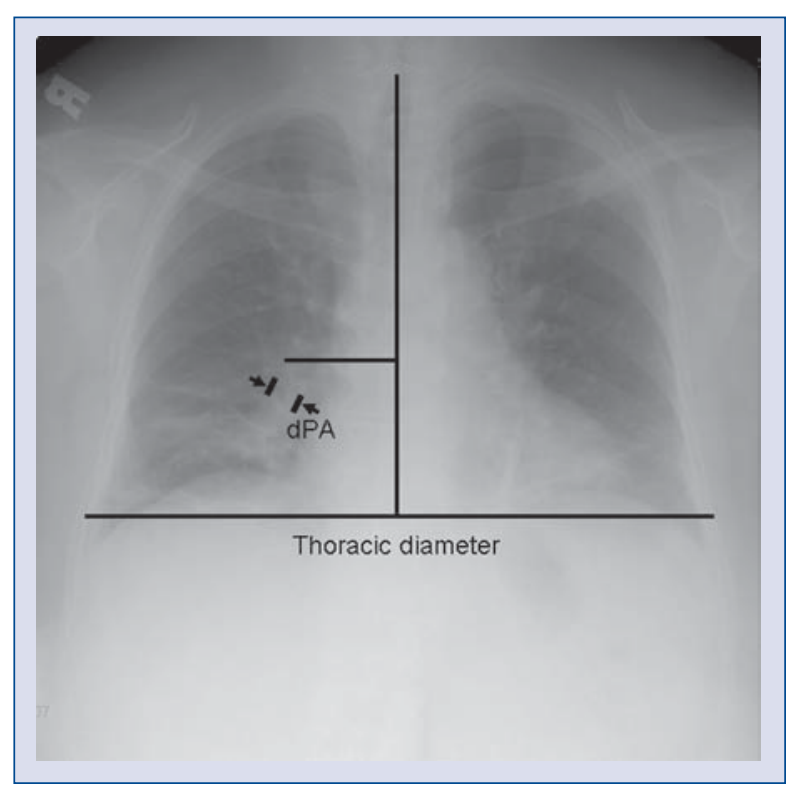

Figure 1. Sites of measurement right descending pulmonary artery (dPA).

\section{Statistical analysis}

The statistical analysis was made with the use of a commercially available statistical package SPSS for Windows, Version 15.0. Continuous variables were expressed as mean \pm standard deviation while categorical variables were expressed as ratios. Categorical and discrete variables were compared using the $\chi^{2}$ test. A receiver operating characteristic curve was used to find an optimal cut-off point of diameter of right descending pulmonary artery on chest X-rays for predicting RV dysfunction in PE patients. A Pearson correlation analysis was made to investigate the possible association between RV dysfunction and diameter of right descending pulmonary artery on chest X-rays. Linear regression analysis was used for defining predictors of RV dysfunction. Probability values of $<0.05$ were considered significant.

\section{Results}

A total of 89 subjects were enrolled in this study. The mean age of the patients was $65 \pm$ \pm 13.5 years with 41 male patients. Fifty $(56.2 \%)$ of the PE patients had RV dysfunction. The frequency of RV dysfunction was significantly higher in patients with an enlarged right descending pulmonary artery on chest X-rays ( $\mathrm{p}=$ $=0.001)$ (Fig. 2, Table 1). There was a significant positive correlation between the diameter of the

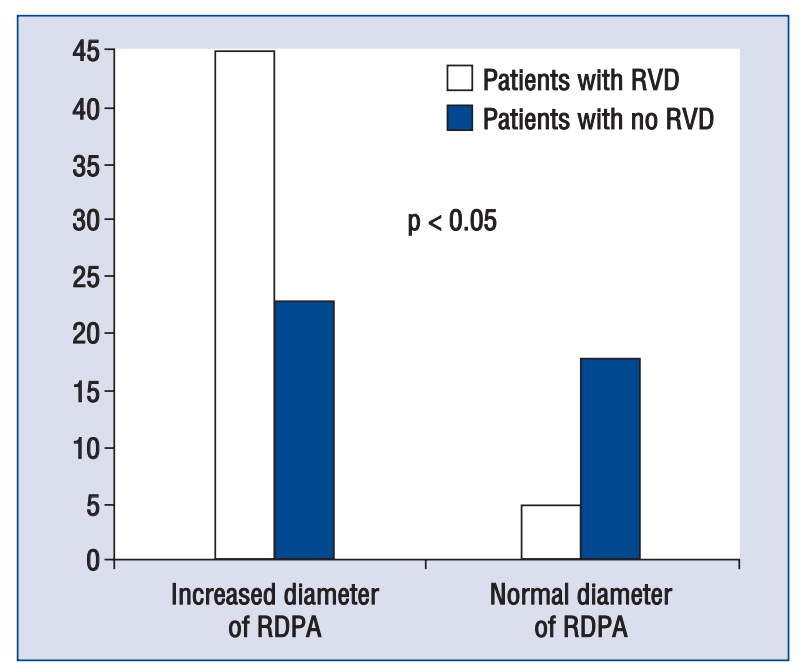

Figure 2. Increased frequency of right ventricular dysfunction (RVD) in hemodynamically stable pulmonary embolism patients with an enlarged right descending pulmonary artery (RDPA) on chest $\mathrm{X}$-rays.

Table 1. Presence of right ventricular dysfunction in hemodynamically stable pulmonary embolism patients with an enlarged right descending pulmonary artery on chest X-rays.

\begin{tabular}{lcc}
\hline $\begin{array}{l}\text { Right ventricu- } \\
\text { lar dysfunction }\end{array}$ & $\begin{array}{c}\text { Diameter of right descending } \\
\text { pulmonary artery on } \\
\text { chest-X-rays }\end{array}$ \\
\cline { 2 - 3 } & Normal & Enlarged \\
\hline Present & 5 & 45 \\
Absent & 18 & 21 \\
Total & 23 & 66 \\
\hline
\end{tabular}

$\chi^{2}: p=0.001$

right descending pulmonary artery on PA chest $\mathrm{X}$-ray and both the RV diameter $(\mathrm{r}=0.469 ; \mathrm{p}=$ $=0.002)$ and the $\mathrm{RV} / \mathrm{LV}$ ratio of end diastolic diameter $(r=0.646 ; p<0.001)$ (Figs. 3, 4). An ROC analysis was used to identify the optimal cut-off value of right descending pulmonary artery diameter as measured on chest X-rays for the prediction of RV dysfunction of PE patients. The AUC was 0.76 for defining RV dysfunction on the basis of the diameter of the right descending pulmonary artery on chest X-rays with a sensitivity of $78 \%$ and a specificity $63 \%$ at the diameter of $16.25 \mathrm{~mm}$ (Fig. 5). When RV dysfunction was predicted it was found that diameter of the right descending pulmonary artery on chest X-rays (Beta $=0.26$; $\mathrm{p}=0.024)$, systolic pulmonary arterial pressure 


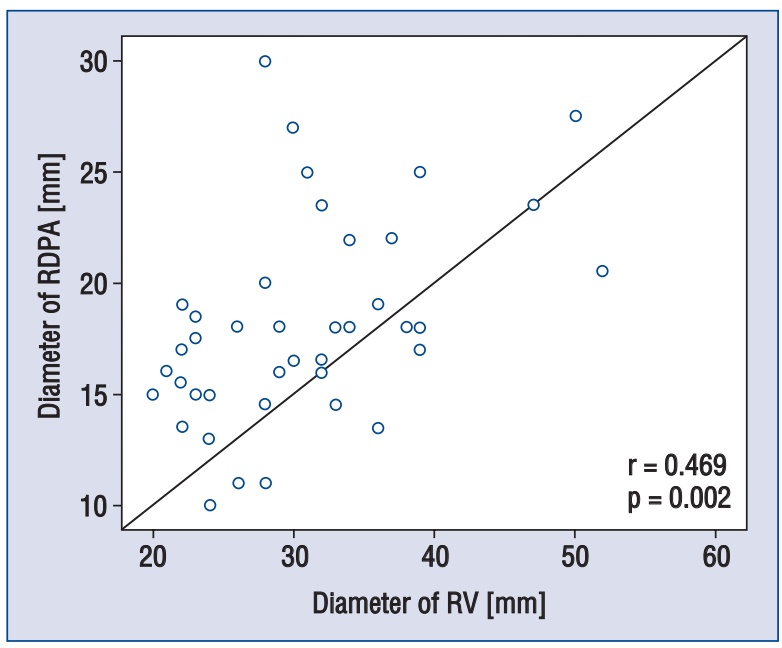

Figure 3. Correlation between diameter of right descending pulmonary artery (RDPA) on postero-anterior chest $\mathrm{X}$-ray and right ventricular (RV) diameter on echocardiography.

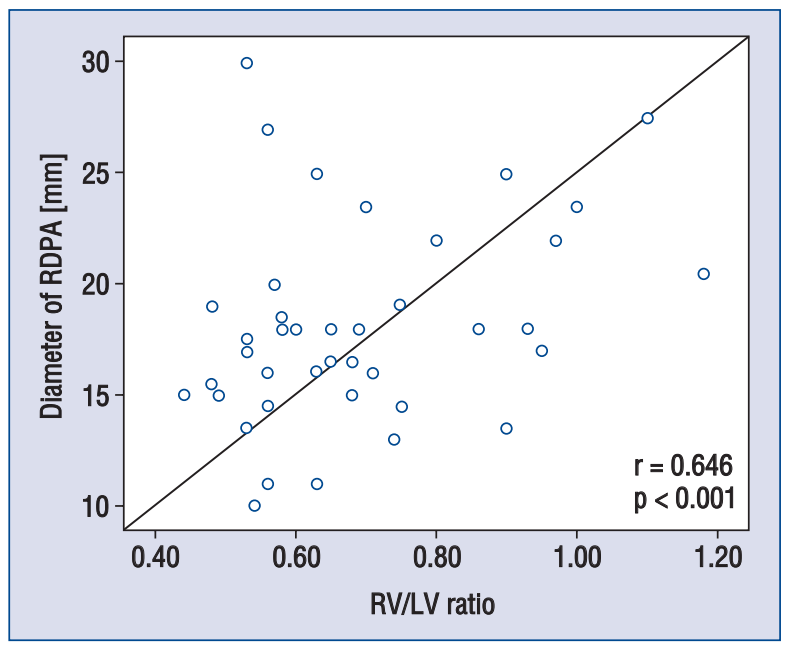

Figure 4. Correlation between diameter of right descending pulmonary artery (RDPA) on postero-anterior chest $\mathrm{X}$-ray and right ventricular/left ventricular (RV/LV) ratio of end diastolic diameter on echocardiography.

on echocardiography $($ Beta $=0.48 ; \mathrm{p}=0.001)$ and troponin-T levels $($ Beta $=0.30 ; \mathrm{p}=0.012)$ were significant predictors with linear regression analysis (Table 2).

\section{Discussion}

Risk stratification in hemodynamically stable $\mathrm{PE}$ has been gaining importance recently. RV dysfunction detected by echocardiography or by biomarkers including especially troponin-T, BNP and

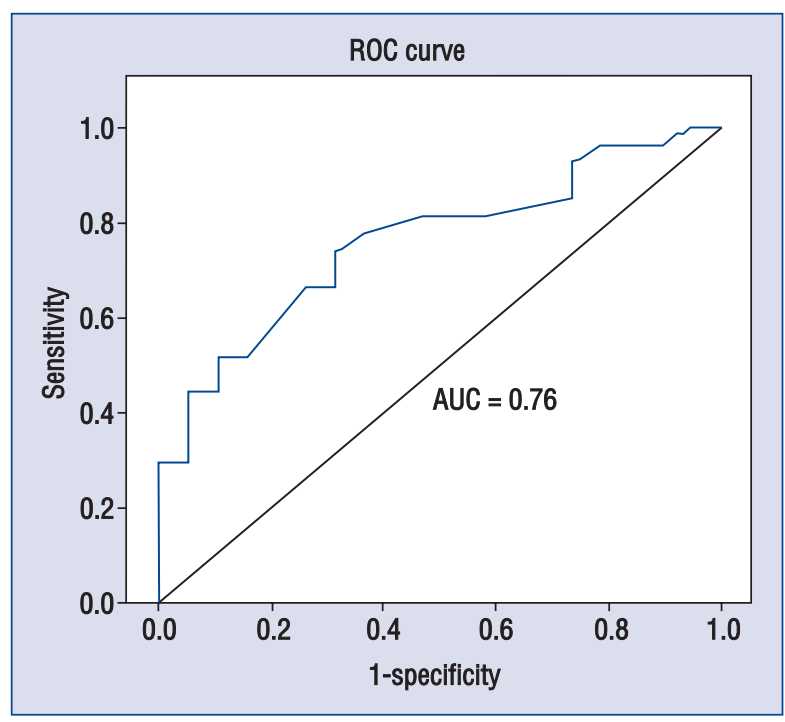

Figure 5. Receiver operating curve analysis to identify the optimal cut-off value of right descending pulmonary artery diameter on chest X-rays for the prediction of right ventricular dysfunction by echocardiography in hemodynamically stable pulmonary embolism patients.

Table 2. Predictors of right ventricular dysfunction in hemodynamically stable pulmonary embolism patients with linear regression analysis.

\begin{tabular}{lcc}
\hline $\begin{array}{l}\text { Predictors of right } \\
\text { ventricular } \\
\text { dysfunction }\end{array}$ & $\begin{array}{c}\text { Standardized } \\
\text { coefficients } \\
\text { Beta }\end{array}$ & $\mathbf{P}$ \\
\hline $\begin{array}{l}\text { Diameter of right } \\
\text { descending } \\
\text { pulmonary artery on } \\
\text { chest-X-rays }\end{array}$ & 0.26 & 0.024 \\
$\begin{array}{l}\text { Systolic pulmonary } \\
\text { arterial pressure on } \\
\text { echocardiography }\end{array}$ & 0.48 & 0.001 \\
\begin{tabular}{l} 
Troponin-T levels \\
\hline
\end{tabular} & 0.30 & 0.012 \\
\hline
\end{tabular}

NT-proBNP has been known to be associated with a poor prognosis in PE [6, 19, 22, 23]. However, echocardiography and these biomarkers are not always available to determine the prognosis in acute $\mathrm{PE}$, especially in acute settings. Simple and easily applied parameters for identifying RV dysfunction in $\mathrm{PE}$ are needed. The present study showed that increased diameter of the right descending pulmonary artery on chest X-rays is a simple and easily applied measure that has a significant correlation with RV dysfunction on echocardiography in hemodynamically stable PE patients. Both RV diameter and the end-diastolic $\mathrm{RV} / \mathrm{LV}$ ratio had a significant 
association with the diameter of right descending pulmonary artery on chest X-rays. An enlarged right descending pulmonary artery was matched in patients with confirmed and unconfirmed PE and has been more frequently seen in embolic patients in literature [24]. Previously it has also been shown that there is an association between the diameter of the right descending pulmonary artery and pulmonary hypertension as revealed by right heart catheterization and by echocardiography [17, 25]. If the diameter of the right descending pulmonary artery as less than $14 \mathrm{~mm}$ was used as a cut-off value, it was found that the accuracy of diagnosis of the mean pulmonary arterial pressure as measured by right heart catheterization lower than $20 \mathrm{~mm} \mathrm{Hg}$ was $69.23 \%$ [25]. With the cut-off point of the diameter of the right descending pulmonary artery as $21 \mathrm{~mm}$ it was shown that specificity for echocardiographic pulmonary hypertension was $97.9 \%$ [17]. Palla et al. [26] found that the radiographic width of the descending pulmonary artery enabled the clinician to identify patients most seriously ill, since it showed a positive linear correlation with the degree of perfusion impairment. The RV dysfunction with defined echocardiographical parameters has not been compared with the right descending pulmonary artery on chest X-rays previously. The present study showed that the diameter of right descending pulmonary artery on chest X-ray had a sensitivity of $78 \%$ and a spesificity of $63 \%$ at the diameter of $16.25 \mathrm{~mm}$ for defining echocardiograhic $\mathrm{RV}$ dysfunction in PE patients. The present study demonstrated that an increased diameter of the right descending pulmonary artery on chest X-ray in acute $\mathrm{PE}$ patients may predict echocardiographic $\mathrm{RV}$ dysfunction in these patients. An association between RV hypokinesis and right descending pulmonary artery has been studied previously and chest radiographs were interpreted to show pulmonary artery enlargement for 118 of 309 patients with RV hypokinesis (sensitivity, 0.38) and for 117 of 483 patients without RV hypokinesis (specificity, 0.76) [15]. However other parameters of RV dysfunction including dilatation of the RV (diastolic diameter $>30 \mathrm{~mm}$ ), abnormal motion of the interventricular septum, tricuspid valve regurgitation (jet velocity $>2.5 \mathrm{~m} / \mathrm{s}$ ) and end diastolic RV/LV ratio other than RV hypokinesis were not studied to investigate the possible association between the diameter of the right descending pulmonary artery on chest X-rays with a defined cut-off point and RV dysfunction on echocardiography. Chest X-rays are already easily applied and performed in all emergency settings especially at the beginning of diagnosis of PE. Diameter of right descending pulmonary artery on chest X-rays was found as a significant predictor of RV dysfunction besides the commonly used troponin-T levels and systolic pulmonary arterial pressure in the present study. Therefore measurement of the diameter of the right descending pulmonary artery on chest $\mathrm{X}$-ray can be a simple method for detecting echocardiographic RV dysfunction in PE patients. The changes in diameter of the right descending pulmonary artery in $\mathrm{PE}$ are known and these changes are attributed to the acute occlusion of some part of the pulmonary vascular bed, producing a rise of pulmonary vascular resistance and pressure [16, 27]. Increased pulmonary vascular resistance has a potential to be associated with RV dysfunction in PE [28]. Through all these mechanisms the changes in the diameter of right descending pulmonary artery may be indirectly associated with the RV dysfunction that is gold standard for the prognosis of PE. The present study has shown that $\mathrm{RV}$ dysfunction is more frequently seen in patients with an increased diameter of the right descending pulmonary artery on chest X-ray and that this was statistically significant.

\section{Limitations of the study}

There are several certain limitations to the present study. The sample size of the present study is small because we did not include PE patients without having RV echocardiographic measurements within $24 \mathrm{~h}$ of admission into the study. PE patients with technical difficulties about reading of PA chest X-rays were not also included into the study which was another reason for the small sample size. Secondly, this is not a follow-up study to compare the mortality or re-embolism of PE patients who had an enlarged diameter of the right descending pulmonary artery on chest $\mathrm{X}$ rays. Thirdly, we did not exactly know whether chest $\mathrm{X}$ rays were performed in the upright or in the supine position for the standardization due to retrospective feature of the study.

\section{Conclusions}

In conclusion, hemodynamically stable PE patients with greater diameter of the right descending pulmonary artery on chest X-rays have more frequent RV dysfunction. There is a correlation between RV dysfunction parameters on echocardiography and the diameter of the right descending pulmonary artery on chest X-rays in hemodynamically stable PE patients. The suggested cut-off point of 
the diameter of the right descending pulmonary artery on chest X-rays is 16.25 to predict RV dysfunction in hemodynamically stable PE patients. Diameter of right descending pulmonary artery on chest X-rays may be used as a predictor of RV dysfunction besides the commonly used troponin-T levels and systolic pulmonary arterial pressure. The diameter of the right descending pulmonary artery on chest X-rays may help in the risk stratification of hemodynamically stable $\mathrm{PE}$ patients and may be used as a prognostic tool for the appropriate therapeutic management of acute $\mathrm{PE}$ as being an easily applicable radiological parameter.

\section{Acknowledgements}

The authors thank Prof. R.W. Guillery from Oxford University for the English corrections of the manuscript.

\section{Author contributions}

Dr Abul: Contributed to study concept and design, acquisition of data, analysis and interpretation of data, statistical analysis, critical revision of the manuscript for important intellectual content, and drafting of the manuscript; $\mathrm{Dr} \mathrm{Ozsu}$ : Contributed to study concept and design, acquisition of data, analysis and interpretation of data, statistical analysis, critical revision of the manuscript for important intellectual content, and drafting of the manuscript; Dr Karakurt: Analysis of the results, and interpretation of data; $\mathrm{Dr}$ Ozben: Collection of data and interpretation of data; Dr Durmus: Collection of data and interpretation of data; $D$ r. Toprak: Interpretation of data; $\mathrm{Dr} O z l u$ : Coordination of the study, interpretation of the study; Dr Celikel: Interpretation of the study and review before publication.

\section{Conflict of interest: none to declare}

\section{References}

1. Kasper W, Konstantinides S, Geibel A, Tiede N, Krause T, Just H. Prognostic significance of right ventricular afterload stress detected by echocardiography in patients with clinically suspected pulmonary embolism. Heart, 1997; 77: 346-349.

2. Goldhaber SZ, Haire WD, Feldstein ML et al. Alteplase versus heparin in acute pulmonary embolism: Randomised trial assessing right-ventricular function and pulmonary perfusion. Lancet, 1993; 341: 507-511.

3. Ribeiro A, Lindmarker P, Johnsson H, Juhlin-Dannfelt A, Jorfeldt L. Pulmonary embolism: One-year follow-up with echocardiography Doppler and five-year survival analysis. Circulation, 1999; 99: 1325-1330 .

4. Jimenez D, Diaz G, Molina J et al. Troponin i and risk stratification of patients with acute nonmassive pulmonary embolism. Eur Respir J, 2008; 31: 847-853.

5. Conroy S, Kamal I, Cooper J. Troponin testing: Beware pulmonary embolus. Emerg Med J, 2004; 21: 123-124.
6. Pruszczyk P, Kostrubiec M, Bochowicz A et al. N-terminal pro-brain natriuretic peptide in patients with acute pulmonary embolism. Eur Respir J, 2003; 22: 649-653.

7. ten Wolde M, Tulevski, II, Mulder JW et al. Brain natriuretic peptide as a predictor of adverse outcome in patients with pulmonary embolism. Circulation, 2003; 107: 2082-2084.

8. Kaczynska A, Pelsers MM, Bochowicz A, Kostrubiec M, Glatz JF, Pruszczyk P. Plasma heart-type fatty acid binding protein is superior to troponin and myoglobin for rapid risk stratification in acute pulmonary embolism. Clin Chim Acta, 2006; 371: 117-123.

9. Lankeit M, Kempf T, Dellas C et al. Growth differentiation factor-15 for prognostic assessment of patients with acute pulmonary embolism. Am J Respir Crit Care Med, 2008; 177: 1018-1025.

10. Abul Y, Karakurt S, Ozben B, Toprak A, Celikel T. C-reactive protein in acute pulmonary embolism. J Investig Med, 2011; 59: 8-14.

11. Figley MM, Gerdes AJ, Ricketts HJ. Radiographic aspects of pulmonary embolism. Semin Roentgenol 1967;2:389-405.

12. Palla A, Petruzzelli S, Donnamaria V, Rossi G, Maltinti G, Giuntini C. Radiographic assessment of perfusion impairment in pulmonary embolism. Eur J Radiol, 1985; 5: 252-255.

13. Weidner W, Swanson L, Wilson G. Roentgen techniques in the diagnosis of pulmonary thromboembolism. Am J Roentgenol Radium Ther Nucl Med, 1967; 100: 397-407.

14. Talbot S, Worthington BS, Roebuck EJ. Radiographic signs of pulmonary embolism and pulmonary infarction. Thorax, 1973; 28: 198-203.

15. Elliott CG, Goldhaber SZ, Visani L, DeRosa M. Chest radiographs in acute pulmonary embolism. Results from the international cooperative pulmonary embolism registry. Chest, 2000; 118: 33-38.

16. Palla A, Donnamaria V, Petruzzelli S, Rossi G, Riccetti G, Giuntini C. Enlargement of the right descending pulmonary artery in pulmonary embolism. Am J Roentgenol, 1983; 141: 513-517.

17. Lin SC, Chen RJC, Lee JH. The correlation between right descending pulmonary artery diameter and echocardiography-estimated systolic pulmonary artery pressure. Acta Cardiol Sin, 2009; 25: 213-217.

18. Bush A, Gray H, Denison DM. Diagnosis of pulmonary hypertension from radiographic estimates of pulmonary arterial size. Thorax, 1988; 43: 127-131.

19. Kasper W, Konstantinides S, Geibel A et al. Management strategies and determinants of outcome in acute major pulmonary embolism: Results of a multicenter registry. J Am Coll Cardiol, 1997; 30: 1165-1171.

20. Chang $\mathrm{CH}$. The normal roentgenographic measurement of the right descending pulmonary artery in 1,085 cases. Am J Roentgenol Radium Ther Nucl Med, 1962; 87: 929-935.

21. Kanemoto N, Furuya H, Etoh T, Sasamoto H, Matsuyama S. Chest roentgenograms in primary pulmonary hypertension. Chest, 1979; 76: 45-49.

22. Kucher N, Printzen G, Goldhaber SZ. Prognostic role of brain natriuretic peptide in acute pulmonary embolism. Circulation, 2003; 107: 2545-2547.

23. Kucher N, Rossi E, De Rosa M, Goldhaber SZ. Prognostic role of echocardiography among patients with acute pulmonary embolism and a systolic arterial pressure of $90 \mathrm{~mm} \mathrm{Hg}$ or higher. Arch Intern Med, 2005; 165: 1777-1781.

24. Donnamaria V, Palla A, Petruzzelli S, Manganelli D, Baldi S, Giuntini C. A way to select on clinical grounds patients with high risk for pulmonary embolism: A retrospective analysis in a nested case-control study. Respiration, 1995; 62: 201-204.

25. Viamonte M, Jr., Parks RE, Barrera F. Roentgenographic prediction of pulmonary hypertension in mitral stenosis. Am J Roentgenol Radium Ther Nucl Med, 1962; 87: 936-947.

26. Palla A, Petruzzelli S, Donnamaria V, Rossi G, Mazzotti C, Giuntini C. Clinical significance of the enlargement of the descending pulmonary artery in pulmonary embolism. Radiol Med, 1985; 71: 26-30.

27. McIntyre KM, Sasahara AA. The hemodynamic response to pulmonary embolism in patients wihout prior cardiopulmonary disease. Am J Cardiol, 1971; 28: 288-294.

28. Coutance G, Cauderlier E, Ehtisham J, Hamon M. The prognostic value of markers of right ventricular dysfunction in pulmonary embolism: A meta-analysis. Crit Care, 2011; 15: R103. 